\title{
Common Basal Concept in Primary Care Medicine and Psychosomatic Medicine
}

\section{Hiroshi Bando*}

Department of Medical Research, Tokushima University, Tokushima, Japan

Keywords: Bio-psycho-social; Psychosomatic medicine; Japanese Primary Care Association; Japanese Society of Psychosomatic Internal Medicine; Hinohara-ism

Abbreviation: PSM: Psychosomatic medicine; JPCA: Japanese Primary Care Association; JSPM: Japanese Society of PSM; JSPIM: Japanese Society of Psychosomatic Internal Medicine; WONCA: World Organization of Family Doctors

Primary care medicine has been crucial field for its principle and characteristic functions. Initially, five items were introduced by Institute of Medicine (IOM) in 1978. They are known widely, including Accessibility, Comprehensiveness, Coordination, Continuity and Accountability (ACCCA) [1]. After that, Professor Saultz proposed 5 kinds of cares, which are Access to Care, Comprehensive Care, Coordination of Care, Continuity of Care and Contextual Care (ACCCC) [2]. Furthermore, Starfield's four principles are known as First contact, Longitudinality, Comprehensiveness and Coordination [3]. From mentioned above, the concept becomes the basis and the practical framework involves the structure, the process and the outcome.

There is a meaningful history concerning the development of primary care. People have hoped ideal doctors who can take care physically and psychologically [4]. Medical practice has developed for long years, but there were growing concern about the necessity for increasing comprehensive and continuous patient care [5]. In 1966, there was a famous report from the Citizens Commission on graduate medical education, which was Millis report [6]. It showed the tendency of fragmentation of care and depersonalization of medicine and recommended the necessity of a physician who can manage the patient from the holistic aspect. The percentage of family physician potential in every 9 years from 1931 to 1957 were $75 \%, 67 \%, 55 \%, 45 \%$, respectively, indicating acute decreasing of family doctors [7]. After that, Family practice was initiated as a new specialty from the Specialty American Boards.

In the light of medical practice quality in primary care, IOM presented six aims for the improvement of health care system function. They are safe, effective, patient centered, timely, efficient, equitable [8]. Among them, patient centered is the key. Furthermore, the committee offered 10 guiding rules concerning patient-clinician relationships for the $21^{\text {st }}$ century. Out of these ten, especially 3 points are important, which are 1. Care based on continuous healing relationships, 3. The patient is the source of control and 6. Safety as a system property [8]. Thus, primary care has the direction of patient centered management. In other words, primary care would include holistic point of view associated with taking care of the body and soul as a human being.

Primary care medicine has developed in various manner in each country. They are Family medicine in United States, general practice in European countries and general internal medicine in Japan.

In the case of Japan, Primary care medicine was introduced and launched by Dr. Hinohara who was the president emeritus of Saint Lukes' International Hospital, Tokyo. He has developed primary care for long period [9]. Then, he has been called "the father of Primary
Care in Japan." His contribution also brought the current activities of Japanese Primary Care Association (JPCA).

Japan has long history and characteristic oriental culture, associated with feeling love for all creatures and nature environment. Taking these into consideration, Dr. Hinohara often emphasize the importance of "the combination of mind and body". Consequently, he developed not only primary care medicine, but also psychosomatic medicine in Japan.

There are common important basal philosophy in primary care and psychosomatic medicine (SPM). These factors are the concept of interrelationship of mind and body, bio-psycho-social points of view and so on [10]. Health in the primary care has been influenced by not only biological, but also psychological and social factors. They include multi-morbidity of some diseases, morbidity burden, patient's complexity and other health-related individual attributes [11].

In the daily medical practice of primary care, there are various health problems. Among them, one of the interesting problems would be Medically Unexplained Symptoms (MUS) [12]. This has other medical terms, such as Somatic Symptom Disorders (SSD), Functional Somatic Symptoms (FSS), bodily-distress syndrome, somatic symptom distress and so on. There are several discussion and controversy concerning MUS and related situations, because these have ambiguity of symptom and diagnosis in actual clinical practice $[13,14]$.

According to several data, MUS has been observed about 10-15\% out of all consultations in GP office [15]. Other reports with the study of clinical prevalence of FSS showed approximately from $9 \%$ to $30 \%$ [16,17]. It seems rather difficult to show precise and correct ratio of MUS, because there are a variety of physical and psychosomatic problems in the daily primary care practice. Consequently, clinical study will be expected in the overlapped field of primary care and psychosomatic medicine.

As regard to psychosomatic medicine, its basic portion exist not in the psychiatry but in the internal medicine. It can also treat patients in the psychological aspect carefully as we as in the physical aspect. The Japanese Society of PSM (JSPM) was established in 1959 and the first Department of Psychosomatic Internal Medicine in the university was started in 1963 [18]. PSM in Japan has revealed a prominent, unique development. It is comprised from medical doctors such as Psychosomatic Internal Medicine (PIM) specialists, general internists, psychiatrists, pediatricians, obstetricians and gynecologists, dentists, dermatologists and others [10].

${ }^{*}$ Corresponding author: Hiroshi Bando, MD, PhD, FACP, Department of Medical Research, Tokushima University, Tokushima, Japan, Tel: +81-90-3187-2485; E-mail: pianomed@bronze.ocn.ne.jp

Received: October 22, 2018; Accepted: December 10, 2018; Published: December 17, 2018

Citation: Bando H (2018) Common Basal Concept in Primary Care Medicine and Psychosomatic Medicine. Prim Health Care 8: 318. doi: 10.4172/21671079.1000318

Copyright: (c) 2018 Bando $\mathrm{H}$. This is an open-access article distributed under the terms of the Creative Commons Attribution License, which permits unrestricted use, distribution, and reproduction in any medium, provided the original author and source are credited. 
Along with the development of JSPM, Japanese Society of Psychosomatic Internal Medicine (JSPIM) was founded in 1996. It is another major society with mainly composed of internists [19]. After that, the first joint congress of five major PSM associations from each field was held in 2009. They included the Japanese Society of Psychosomatic Medicine, Psychosomatic Obstetrics and Gynecology, Psychosomatic Pediatric Medicine, Psychosomatic Dental Medicine and Psychosomatic Internal Medicine. Furthermore, several subdivided societies have been established for cardiovascular, digestive, dermatological and oriental medicine [20].

JSPM and JSPIM participate in international activities including publishing BioPsychoSocial Medicine (BPSM) [10]. It plays a crucial role in continuing research in psychosomatic medicine, especially focusing on mind-body relationships [21]. It covers all fields such as behavioral and social sciences, neuroscience, stress physiology and epidemiology, psycho-neuro-endocrinology/immunology and psychooncology, all of which are associated with mind-body interactions [21].

PSM in Japan has continued practice and research on psychotherapies, including transactional analysis, autogenic therapy and cognitive behavioral therapy. For mutual interrelationship, Japanese Union of Associations for Psycho-medical Therapy (UPM) has promoted various activities [10].

Michael Balint emphasized "the necessity to cultivate communication between patients and physicians for better treatment from the biopsychosocial point of view" [22] . Furthermore, primary care physicians with PSM skills compared to psychiatrists can manage variety of health problems. In the near future, systematic cooperation between primary care and PSM would be expected to develop with mutual understanding and cooperation.

In summary, primary care and psychosomatic medicine have close relationship in the light of the bio-psycho-social model system [23]. For years, psychosomatic medicine in primary care has been effected by the physicians and psychoanalysts who emulated Balint's approach for psychosomatic and holistic perspectives [22]. Furthermore, the philosophy of Hinohara-ism would be involved in the basal concept [24]. We would expect the development of practice and research of these field for the wellness and happiness of the people.

\section{References}

1. Institute of medicine (1978) A manpower policy for primary health care: Report of a study. Washington, DC: The National Academies Press, pp: 1-120.

2. Saultz JW (2001) Textbook of family medicine. McGraw-Hill, pp: 1-830.

3. Starfield B (1998) Primary care: Balancing health needs, services, and technology. Oxford University Press, pp: 1-438.
4. Rutstein DD (1900) Do you really want a family doctor? Harpers (N Y N Y) 221 144-150.

5. Fahs IJ, Peterson OL (1968) The decline of general practice. Public Health Rep 83: 267-270.

6. Millis JS (1967) The graduate education of physicians. N Engl J Med 276:11011104.

7. Overpeck MD (1970) Physicians in family practice 1931-67. Public Health Rep 85: $485-494$

8. Wolfe A (2001) Institute of medicine report: Crossing the quality chasm: A new health care system for the 21st century. Policy Polit Nurs Pract 2: 233-235.

9. Bando H, Yoshioka A, Iwashimizu Y, Iwashita M, Doba N (2017) Development of primary care, lifestyle disease and new elderly association (NEA) in Japancommon philosophy with Hinohara-ism. Prim Health Care 7: 281.

10. Murakami M, Nakai $Y$ (2017) Current state and future prospects for psychosomatic medicine in Japan. Biopsychosoc Med 11: 1-10.

11. Pizzini A, Marrocco W, PioD'Ingianna A, Marri G (2017) The biopsychosocial vulnerability in primary care. J Health Sci 5: 159-165.

12. Rosendal M, Carlsen AH, Rask MT, Moth G (2015) Symptoms as the main problem in primary care: A cross-sectional study of frequency and characteristics. Scand J Prim Health Care. 33: 91-99.

13. Creed F, Guthrie E, Fink P, Henningsen P, Rief W, et al. (2010) Is there a better term than "medically unexplained symptoms"? J Psychosom Res 68: 5-8.

14. Marks EM, Hunter MS (2015) Medically unexplained symptoms: An acceptable term? Br J Pain. 9: 109-114.

15. Brown RJ (2007) Introduction to the special issue on medically unexplained symptoms: Background and future directions. Clin Psychol Rev 27: 769-780.

16. Eikelboom EM, Tak LM, Roest AM, Rosmalen JGM (2016) A systematic review and meta-analysis of the percentage of revised diagnoses in functional somatic symptoms. J Psychosom Res 88: 60-67.

17. Graver CJ (2017) Functional somatic syndrome: Assessment and management J Am Osteopath Assoc 117: 511-519.

18. Ikemi $Y$ (1993) Psychosomatic medicine as a link of the west and the east. Jpn J Psychosom Med 33: 546-552.

19. Japanese Society of Psychosomatic Internal Medicine (2016) Journal of the 20th Anniversary. Jpn J Psychosom Int Med 20: 2.

20. Komaki G (2018) A new milestone for biopsychosocial medicine. Biopsychosoc Med 12: 10 .

21. Deter HC, Kruse J, Zipfel S (2018) History, aims and present structure of psychosomatic medicine in Germany. Biopsychosoc Med 12: 1.

22. Balint M (1964) The Doctor, his Patient and the Illness. Pitman: London.

23. Fritzsche K (2014) Psychosomatic Medicine in Primary Care. In: Fritzsche K, McDaniel S, Wirsching M (Eds.) Psychosomatic Medicine. Springer: New York, NY.

24. Hinohara S, Doba N (2005) The future profile of health promotion and disease prevention in Japan. Methods Inf Med 44: 342-347. 